\title{
Diversity and Distribution of Vascular Epiphytic Flora in Sub-temperate Forests of Darjeeling Himalaya, India
}

\author{
Preshina Rai ${ }^{1}$ and Saurav Moktan ${ }^{1^{*}}$ \\ ${ }^{1}$ Department of Botany, University of Calcutta, 35, B.C. Road, Kolkata, 700 019, West Bengal, India.
}

Authors' contributions

This work was carried out in collaboration between both authors. Author PR conducted field study, collected data and prepared initial draft including literature searches. Author SM provided taxonomic expertise with identification and data analysis. Both authors read and approved the final manuscript.

Article Information

DOI: $10.9734 / A R R B / 2020 / v 35 i 530226$

Editor(s):

(1) Dr. Rishee K. Kalaria, Navsari Agricultural University, India.

Reviewers:

(1) Sameh Cherif, University of Carthage, Tunisia.

(2) Ricardo Moreno-González, University of Göttingen, Germany.

(3) Nelson Túlio Lage Pena, Universidade Federal de Viçosa, Brazil. Complete Peer review History: http://www.sdiarticle4.com/review-history/57913

Original Research Article

Received 06 April 2020

Accepted 11 June 2020

Published 22 June 2020

\section{ABSTRACT}

Aims: This communication deals with the diversity and distribution including host species distribution of vascular epiphytes also reflecting its phenological observations.

Study Design: Random field survey was carried out in the study site to identify and record the taxa. Host species was identified and vascular epiphytes were noted.

Study Site and Duration: The study was conducted in the sub-temperate forests of Darjeeling Himalaya which is a part of the eastern Himalaya hotspot. The zone extends between 1200 to 1850 $\mathrm{m}$ amsl representing the amalgamation of both sub-tropical and temperate vegetation. The study was conducted during the year 2018-2019.

Methodology: Process of random sampling collection was followed. Host trees with $\mathrm{CBH}>30 \mathrm{~cm}$ were identified and percentage of distribution of epiphytes in the host tree was analyzed and divided into two zones depending on the abundance of epiphytes. Data for the epiphytic plant specimens were collected in the field. Exsiccates were made with the collected voucher specimens and were deposited at the Calcutta University Herbarium (CUH) following the conventional methodology. Study on the taxa are based on their host tree distribution, micro-habitat and phenological status. The location and altitude of the study sites were recorded by global positioning system. 
Results: A total number of 115 species under 70 genera and 31 families have been identified with $65 \%$ other vascular angiosperms (basal angiosperms and eudicots) and $16 \%$ monocots. Orchidaceae among the monocots represented $36 \%$ with 41 species and 18 genera. Ferns and lycophytes include $25 \%$ of the diversity with 29 taxa under 6 families. Most favorable host trees were Ficus auriculata Lour., Ficus neriifolia Sm., Saurauia nepalensis DC., Erythrina variegata L., Macaranga denticulata (Blume) Müll. Arg., Cryptomeria japonica (Thunb. ex L.f.) D. Don and Engelhardia spicata Lechen ex Blume.

Conclusion: The other vascular angiosperms preferred wet monsoon for flowering whereas spring season was most favourable for Orchidaceae. Although a healthy number of vascular epiphytes have been identified, a number of threats are still prevailing in the regions which are mostly anthropogenic. Proper collaborative strategies have to be maintained for its conservation.

Keywords: Vascular angiosperm; phenology; orchidaceae; host tree; conservation.

\section{INTRODUCTION}

Originally, the name epiphyte is derived from two Greek words 'epi=upon and phyton=plants' [1]. Epiphyte may be defined as a plant that root and perch on the surface of another plant or non living objects without driving food from its host [25]. They are a unique and diverse group of plants with very interesting ecology [6] comprising about $10 \%$ of the world's total flora [7]. Epiphytes are taxonomically heterogeneous group composed of over 28,000 species under 84 families and represent an important proportion of world flora $[8,9]$. They may be divided into holo-epiphytes, primary hemi-epiphytes, and secondary hemiepiphytes functional types [10]. Holoepiphytes never have root contact with the soil while hemiepiphytes are connected to the soil during part of their life-cycle at which stage they resemble vines (i.e. herbaceous climbers). Primary hemiepiphytes germinate on other plants, usually trees, only to become terrestrially-rooted through aerial roots while secondary hemi-epiphytes germinate in the soil to lose contact later. They contribute to the richness of local and regional floristic diversity Microhabitats on trees trunks, barks and irregular surfaces on branches are all favourable for the growth of epiphytes [11]. Epiphytes ecologically contribute to forest biodiversity in increasing species richness [12], primary productivity, biomass, water retention and also provide substrate for nitrogen fixing bacteria and serve as indicators of change in microclimate as well as global climatic change $[13,14]$ including nutrient store [15]. They also provide food and shelter for fauna [16]. Some angiospermic epiphytes have ethnomedicinal uses and are used against several ailments [17]. Vascular epiphytes are vulnerable group and consequently represent a good indicator group of biodiversity that can be monitored to assess the effects of forest disturbance [18]. Many vascular epiphytes exhibit physiological and morphological characteristics that help them survive drought [19] and also assist in water retention, such as thickened or succulent leaves, or rhizomes and specialized water storage tissue $[4,6,20,21,22]$. The vascular epiphytes are sensitive to moisture which makes them a good environmental indicator for both successional stages of forest ecosystems and natural environmental variations [23,24]. The communities of vascular epiphytes have been used as bio-indicators of climate change, pollution and damage to ecosystems $[25,26,27]$. This sensitivity becomes important in the context of conservation, given that many of the epiphytic species have a high degree of specialization with their phorophyte, thus becoming vulnerable to extinction by habitat and climate change $[28,29]$. Vascular epiphytes in majority comprise of fern and monocots - especially orchids, bromeliads and aroids with relatively fewer other angiosperms [30]. Phorophyte structure, substrate availability and dispersal syndromes are main factors for distribution pattern of vascular epiphytes [31,32]. Besides, establishment of vascular epiphytes provide suitable niche through autogenic succession to other epiphytes [33]. The species of vascular epiphytes are increasingly threatened and decrease in population due to over-collecting horticulturally valuable species for commercial purposes and habitat loss due to deforestation and land use changes [30]. Works related to epiphytes have been conducted in Western Ghats [34-36], Western Himalayas [37-40], Central Himalayas [41-43] as well as towards the Eastern Himalaya [44-47].

Although, several workers have explored the flora of Darjeeling Himalaya [48-54], only few 
notable works with particular reference to vascular epiphytes are known [45,46]. Therefore, there is a need to investigate and understand the rich vascular epiphytic flora from Darjeeling region of eastern Himalaya.

\section{METHODOLOGY}

\subsection{Study Area}

The present study was conducted in the Subtemperate forests of Darjeeling Himalaya. The Darjeeling Himalaya extends between $27^{\circ} 13^{\prime} 10^{\prime \prime}$ $\mathrm{N}$ to $26^{\circ} 27^{\prime} 05^{\prime \prime} \mathrm{N}$ Latitude and $88^{\circ} 53^{\prime} \mathrm{E}$ to $87^{\circ} 59^{\prime}$ $30^{\prime \prime} \mathrm{E}$ Longitude that is located in India's state West Bengal, in the Himalayan foothills at an altitudinal range of 130 to $3636 \mathrm{~m}$ amsl. It is situated at the northernmost end of the Eastern India in the form of an inverted wedge, covering about $3.68 \%$ of the total area of the state of West Bengal. The northernmost point of the district is the tri-junction near Phalut [27 $13^{\prime} 10^{\prime \prime} \mathrm{N}$; $88^{\circ} 21^{\prime} E$ ] and the southern-most point is the Phansidewa block [26 $27^{\prime} 05^{\prime \prime} \mathrm{N}$; 88 $\left.22^{\circ} \quad \mathrm{E}\right]$; likewise the west to east extension of the district lies between Sabarkum near Sandakphu [87 $59^{\prime}$ $30^{\prime \prime} \mathrm{E} ; 27^{\circ} 12^{\prime} \mathrm{N}$ ] and Todey village along river

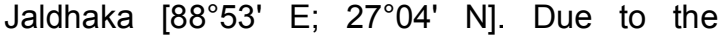
altitudinal variation that range from tropical plain to sub-alpine zone, different climatic zones are available in Darjeeling Himalaya which offers favourable environment for the growth and development of all plant species including epiphytes and their migration in different habitats [52]. The Sub-temperate vegetation zone has been classified between 1200 to $1850 \mathrm{~m}$ amsl and represents the amalgamation of both the sub-tropical and temperate vegetation with intermediate type of forest composition. The climatic set-up of four seasons are recognized in the region: (i) Winter from December to February, (ii) Spring and summer from March to May, (iii) Monsoon or Rainy Season from June to August, and (iv) Autumn from September to November [51]. The temperature in the region varies with a minimum of $2.4^{\circ} \mathrm{C}$ to a maximum of $9.6^{\circ} \mathrm{C}$ during winter, $8.3^{\circ} \mathrm{C}$ to $19.1^{\circ} \mathrm{C}$ during spring and summer and $12^{\circ} \mathrm{C}$ to $18^{\circ} \mathrm{C}$ during autumn season with an average annual precipitation of about 337.3 $\mathrm{mm}$.

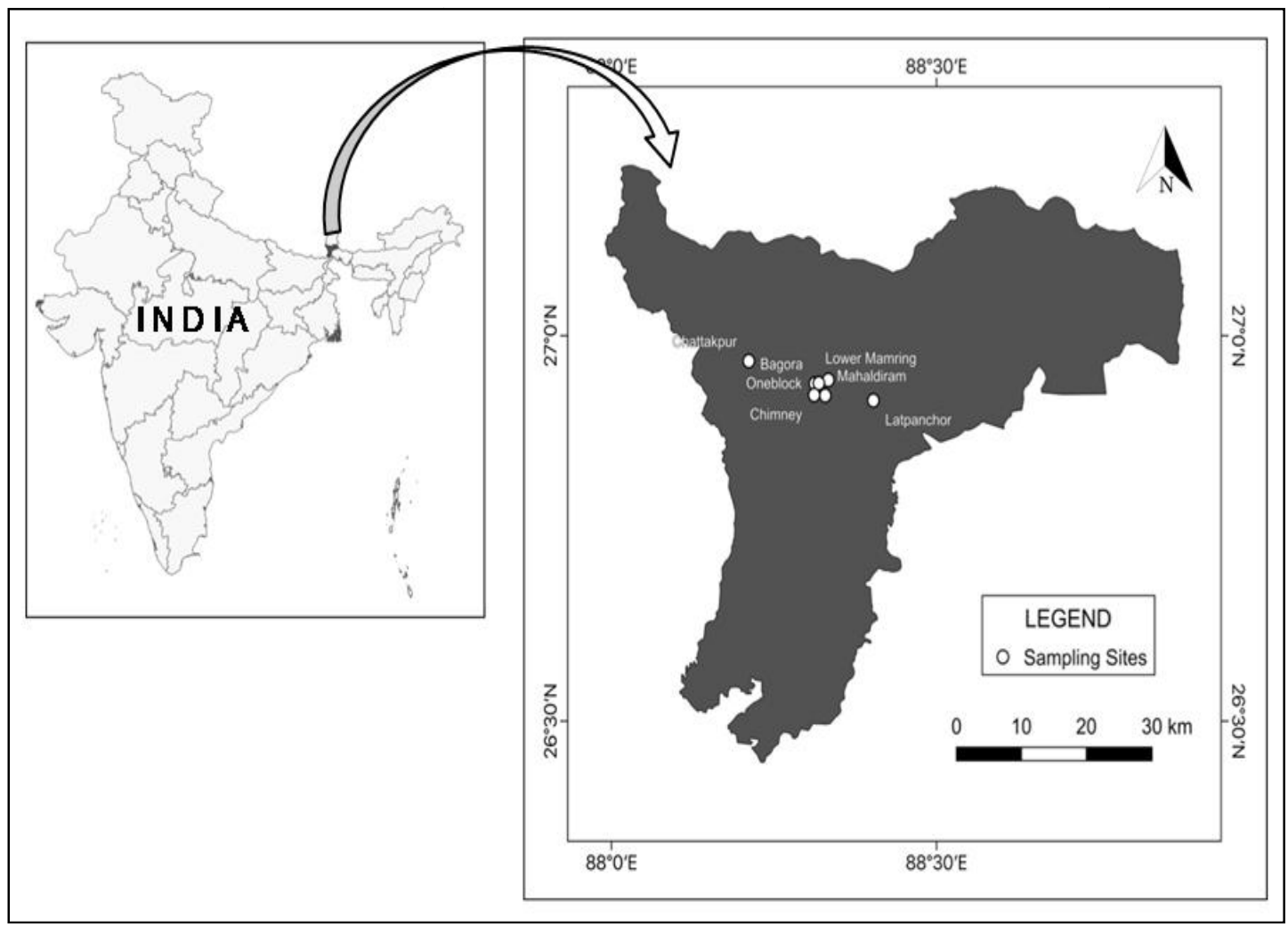

Fig. 1. Map of the study area showing sampling sites 


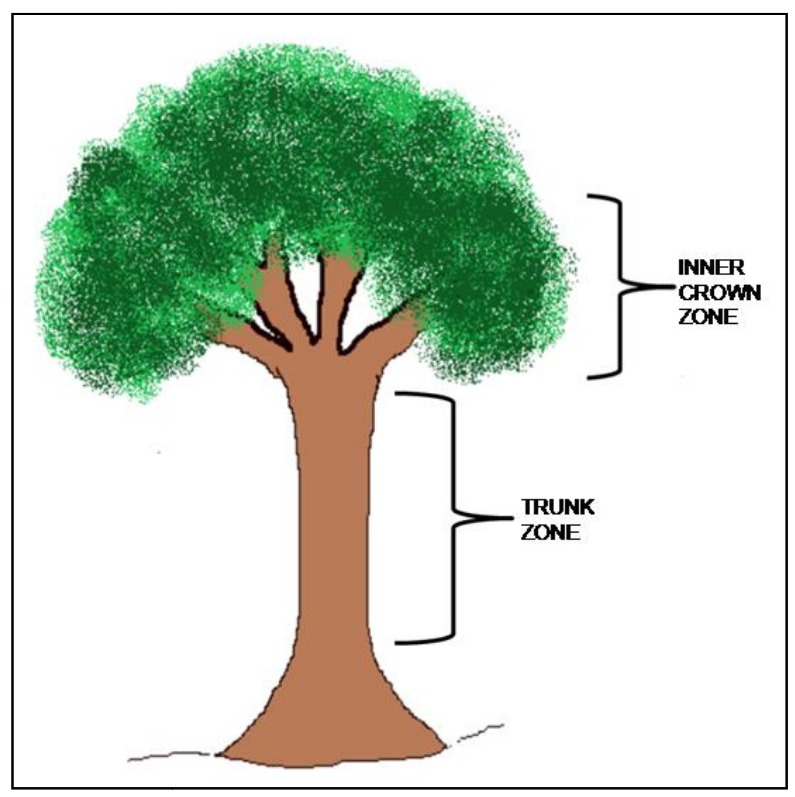

Fig. 2. Zone of host tree trunk

\subsection{Data Collection}

Field survey was carried out from October 2018 to October 2019 in the forest that lied within the Sub-temperate altitude. The process of random sampling collection was followed. Some of the areas under study include Bagora [26 $9378^{\prime} \mathrm{N}$, $88^{\circ} 3127^{\prime} \mathrm{E}$ ], Lower Mamring [26 $56.557^{\prime} \mathrm{N} 88^{\circ}$ 20.018' E], Oneblock [26 $56.302^{\prime} N 8^{\circ} 19.134^{\prime}$

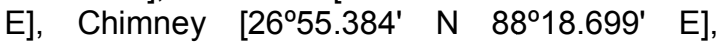

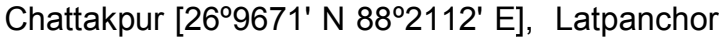

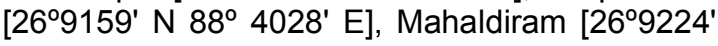
$\mathrm{N} 88^{\circ} 3288^{\prime} \mathrm{E}$ ] and areas where the diversity of vascular epiphytes was rich (Fig. 1).

Data on abundance of epiphytic plant specimens were collected in the field with the assistance of local tree climber; epiphytic plants were collected both from the ground and tree. Host tree species with $>30 \mathrm{~cm}$ CBH were identified and sampled randomly in the forest and segregated into two zones i) Trunk zone covering the area below the first branching till the base and ii) Inner crown zone covering the remaining area above the first branching (Fig. 2). The phenological status of the taxa was also noted through field visit in different seasons. The voucher specimens collected were mounted into herbarium sheets following the conventional methodology [55]. The identification of the taxa were made following suitable literatures and proper nomenclature was maintained following Plants of the World Online [56]. Properly mounted and labelled herbarium exsiccates have been preserved for future study and were deposited at the Calcutta University Herbarium (CUH). The location and altitude of the study sites were recorded by global positioning system (GPS; Garmin eTrexH).

\section{RESULTS AND DISCUSSION}

\subsection{Results}

The present study exposed the interesting composition of vascular epiphytes with a total number of 115 species under 70 genera and 31 families. $81 \%$ were spermatophytic families comprising $65 \%$ other vascular angiosperms that include basal angiosperms and eudicots and 16 $\%$ monocots. The other vascular angiosperms represented 38 species under 28 genera. The dominating family was Ericaceae, Gesneriaceae and Piperaceae with 4 species each followed by Acanthaceae, Apocynaceae and Urticaceae with 3 species (Fig. 3). The monocots were represented by 48 species under 25 genera. Orchidaceae showed biggest abundance on all phorophytes with 18 genera and 41 species representing $36 \%$ with Dendrobium Sw. being the most diverse followed by Bulbophyllum Thouars and Cymbidium Sw. The ferns and lycophytes include $25 \%$ of the taxa identified with 29 species and 17 genera under 6 families with Polypodiaceae being the most abundant family with species like Pyrrosia Mirb. Goniophlebium 
Blume (C. Presl) Oleandra Cav. and Lepisorus (Sm.) Ching (Table 1). The study revealed that the angiospermic herb represented $40 \%$, while the climbers and shrubs were $16(14 \%)$ and 8 (7\%) respectively. Pendulous plants like Hoya serpens Hook.f. and $H$. linearis Wall. ex D.Don also represented $2 \%$ with only $1 \%$ of parasitic plants (Fig. 4).

The vegetation of this short vertical zone between 1200 to $1850 \mathrm{~m}$ was found to be an amalgamation of sub-tropical and temperate type. The zone harbored dominating tree and shrub species like Brassaiopsis hainla (Buch.Ham.) Seem., Brassaiopsis hispida Seem., Engelhardia spicata Lechen ex Blume, Macaranga indica Wight, Alnus nepalensis D. Don, Luculia gratissima (Wall.) Sweet., Buddleja asiatica Lour., Camellia kissi Wall. Casearia glomerata Roxb., Cestrum aurantiacum Lind., Cryptomeria japonica (Thunb. ex L.f.) D. Don, Exbucklandia populnea (R.Br. ex Griff.) R.W.Br., Edgeworthia gardneri (Wall.) Meisn., Boehmeria glomerulifera Miq., B. macrophylla Hornem., Clematis semilacifolia Wall., Porana grandiflora Wall., Tetrastigma rumicispermum (M.A. Lawson) Planch., Osbeckia stellata Buch.-Ham. ex D.Don, Oxyspora paniculata DC., Holboellia latifolia Wall.,, Dichroa febrifuga Lour., Agapetes sikkimensis Airy Shaw. Ground vegetation remains dominated by Persicaria spp, Elatostema spp., Pilea glaberrima (Blume) Blume, Solanum erianthum D. Don, Viola diffusa Ging.

\subsubsection{Distribution within host tree}

The distribution of vascular epiphytes within host tree species with $>30 \mathrm{~cm} \mathrm{CBH}$ was also investigated. The host trees like Ficus auriculata Lour., Ficus neriifolia Sm., Saurauia nepalensis DC., Erythrina variegata L., Macaranga denticulata (Blume) Müll.Arg., Cryptomeria japonica (Thunb. ex L.f.) D. Don, Engelhardia spicata Lechen ex Blume were mostly dominant in epiphytic species richness. The percentage distribution in the host tree crown was also analyzed and it was observed that the number of species significantly decreases from trunk zone to the inner crown zone. The present study showed $74 \%$ of other vascular angiosperms distributed in the trunk zone (TZ) while $26 \%$ towards the inner crown zone (ICZ). In case of monocots, only $2 \%$ were identified from the inner crown zone and the remaining $98 \%$ from the trunk zone especially the Orchidaceae (Fig. 5). About one third of ferns and lycophytes representing $31 \%$ inhabitated the inner crown zone and the maximum taxa (69\%) were distributed on the trunk zone. Host tree species such as Ficus auriculata Lour., Ficus neriifolia Sm., Engelhardia spicata Lechen ex Blume with maximum branching sheltered majority of epiphytes and the distribution was contiguous. Other host trees that include Macaranga denticulata (Blume) Müll. Arg., Cryptomeria japonica (Thunb. ex L.f.) with thin branching sheltered comparatively lesser species.

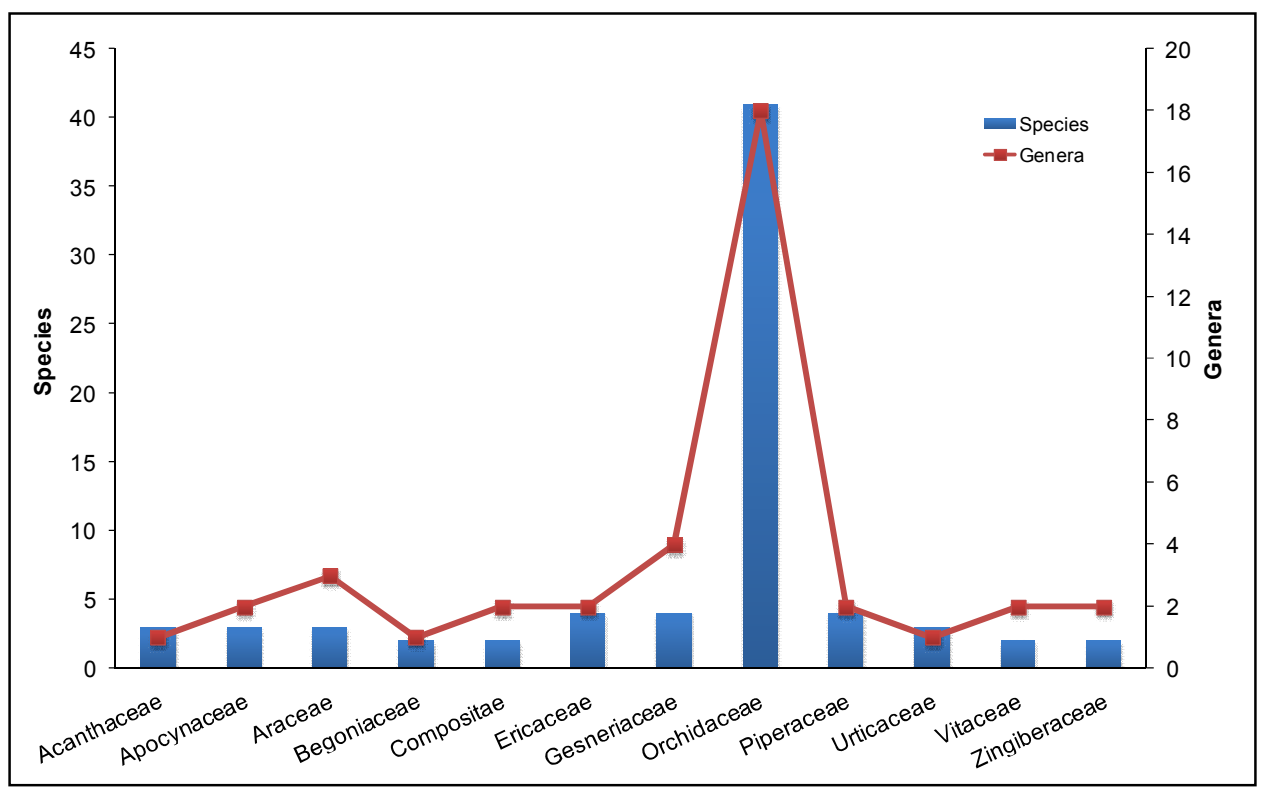

Fig. 3. Dominant families with number of species and genera 
Table 1. Diversity of vascular epiphytes in sub-temperate forests of Darjeeling Himalaya

\begin{tabular}{|c|c|c|c|c|}
\hline Taxa & Habit type & Life form & Distribution & Phenology \\
\hline \multicolumn{5}{|l|}{ Acanthaceae Jussieu } \\
\hline Thunbergia coccinea Wall ex D.Don & $\mathrm{CL}$ & $\mathrm{P}$ & $\mathrm{ICZ}$ & Dec-March \\
\hline Thunbergia indica Alatta & $\mathrm{CL}$ & $\mathrm{P}$ & ICZ & June \\
\hline Thunbergia lutea T. Anderson & $\mathrm{CL}$ & $\mathrm{P}$ & $\mathrm{ICZ}$ & Aug-Dec \\
\hline \multicolumn{5}{|l|}{ Apocynaceae Jussieu } \\
\hline Ceropegia pubescens Wall. & $\mathrm{CL}$ & $\mathrm{P}$ & TZ & July-Sept \\
\hline Hoya linearis Wall. ex D. Don & $\mathrm{Pe}$ & $\mathrm{P}$ & $\mathrm{TZ}$ & Sept \\
\hline Hoya serpents Hook.f. & $\mathrm{Pe}$ & $\mathrm{P}$ & $\mathrm{TZ}$ & July-Oct \\
\hline \multicolumn{5}{|l|}{ Araceae Jussieu } \\
\hline Colocasia affinis Schott & $\mathrm{H}$ & A & TZ & June-Aug \\
\hline Pothos scandens L. & $\mathrm{CL}$ & $\mathrm{P}$ & $\mathrm{TZ}$ & Dec-April \\
\hline Rhaphidophora glauca (Wall.) Schott & $\mathrm{CL}$ & $\mathrm{P}$ & $\mathrm{TZ}$ & Sept-May \\
\hline \multicolumn{5}{|l|}{ Asparagaceae Jussieu } \\
\hline Polygonatum oppositifolium (Wall.)Royle & $\mathrm{H}$ & $\mathrm{P}$ & TZ & May \\
\hline \multicolumn{5}{|l|}{ Asteraceae Berchtold \& J. Presl } \\
\hline Mikania micrantha Kunth & $\mathrm{CL}$ & $\mathrm{P}$ & TZ & June \\
\hline Senecio scandens var. crataegifolius (Hayata)Kitam & $\mathrm{CL}$ & $\mathrm{P}$ & $\mathrm{TZ}$ & Sept-Oct \\
\hline \multicolumn{5}{|l|}{ Begoniaceae C. Agardh } \\
\hline Begonia flaviflora H.Hara & $\mathrm{H}$ & $\mathrm{P}$ & TZ & June-July \\
\hline Begonia hatacoa var. hatacoa Buch-Ham. ex D.Don & $\mathrm{H}$ & $\mathrm{P}$ & $\mathrm{TZ}$ & Oct-Nov \\
\hline \multicolumn{5}{|l|}{ Berberidaceae Jussieu } \\
\hline Holboellia angustifolia Wall. & $\mathrm{CL}$ & A & TZ & April-June \\
\hline \multicolumn{5}{|l|}{ Cucurbitaceae Jussieu } \\
\hline Herpetospermum tonglense (C.B Clarke) H.Schaef. \& S.S Renner & $\mathrm{CL}$ & $\mathrm{P}$ & TZ & June \\
\hline \multicolumn{5}{|l|}{ Dioscoreaceae R. Brown } \\
\hline Dioscorea bulbifera L. & $\mathrm{CL}$ & A & TZ & July-Sept \\
\hline \multicolumn{5}{|l|}{ Ericaceae Durande } \\
\hline Agapetes incurvata (Griff.) & S & $\mathrm{P}$ & ICZ & May-June \\
\hline Agapetes serpens (Wight) Sleumer & $\mathrm{S}$ & $\mathrm{P}$ & ICZ & May-June \\
\hline Vaccinium retusum (Griff.)Hook.f. ex C.B.Clarke & $\mathrm{H}$ & $\mathrm{P}$ & $\mathrm{TZ}$ & June \\
\hline Vaccinium vacciniaceum (Roxb.) Sleumer & $\mathrm{H}$ & $\mathrm{P}$ & $\mathrm{TZ}$ & April \\
\hline
\end{tabular}


Rai and Moktan; ARRB, 35(5): 63-81, 2020; Article no.ARRB.57913

\begin{tabular}{|c|c|c|c|c|}
\hline Taxa & Habit type & Life form & Distribution & Phenology \\
\hline \multicolumn{5}{|l|}{ Gentianaceae Jussieu } \\
\hline Tripterospermum volubile (D.Don) H.Hara & $\mathrm{CL}$ & $\mathrm{P}$ & $\mathrm{TZ}$ & June-Oct \\
\hline \multicolumn{5}{|l|}{ Gesneriaceae Dumortier } \\
\hline Aeschynanthus hookeri C.B.Clarke & $\mathrm{S}$ & $\mathrm{P}$ & ICZ & July-Nov \\
\hline Didymocarpus aromaticus D.Don & $\mathrm{H}$ & $\mathrm{P}$ & $\mathrm{TZ}$ & July-Sept \\
\hline Henckelia pumila (D.Don) A.Dietr. & $\mathrm{H}$ & A & $\mathrm{TZ}$ & Aug \\
\hline Lysionotus serratus D.Don & $\mathrm{S}$ & $\mathrm{P}$ & $\mathrm{TZ}$ & July-Sept \\
\hline \multicolumn{5}{|l|}{ Melastomataceae Jussieu } \\
\hline Oxyspora paniculata DC. & $\mathrm{S}$ & $\mathrm{P}$ & TZ & July-Sept \\
\hline \multicolumn{5}{|l|}{ Moraceae Gaudich } \\
\hline Ficus sarmentosa Buch.Ham. ex Sm. & $\mathrm{CL}$ & $\mathrm{P}$ & $\mathrm{TZ}$ & May-July \\
\hline \multicolumn{5}{|l|}{ Papaveraceae Jussieu } \\
\hline Dactylicapnos scandens (D.Don) Hutch. & $\mathrm{CL}$ & $\mathrm{P}$ & TZ & July-Nov \\
\hline \multicolumn{5}{|l|}{ Piperaceae Giseke } \\
\hline Peperomia heyneana Miq. & $\mathrm{H}$ & $\mathrm{P}$ & TZ & May-Aug \\
\hline Peperomia pellucida (L.) Kunth & $\mathrm{H}$ & $\mathrm{P}$ & $\mathrm{ICZ}$ & April-July \\
\hline Peperomia tetraphylla (G.Forst.) Hook. \& Arn. & $\mathrm{H}$ & $\mathrm{P}$ & $\mathrm{TZ}$ & Feb-April \\
\hline Piper pedicillatum C.DC. & $S$ & $\mathrm{P}$ & $\mathrm{TZ}$ & June \\
\hline \multicolumn{5}{|l|}{ Primulaceae Batsch ex Borkhausen } \\
\hline Lysimachia japonica Thunb. & $\mathrm{H}$ & $\mathrm{P}$ & $\mathrm{TZ}$ & March-April \\
\hline \multicolumn{5}{|l|}{ Rubiaceae Jussieu } \\
\hline Neohymenopogon parasiticus (Wall.) Bennet & $\mathrm{S}$ & $\mathrm{P}$ & ICZ & June-Aug \\
\hline \multicolumn{5}{|l|}{ Santalaceae R. Brown } \\
\hline $\begin{array}{l}\text { Dendrotrophe granulata (Hook.f. \& Thomson ex A.DC) A.N. Henry \& } \\
\text { B.Roy }\end{array}$ & $\mathrm{Pa}$ & $\mathrm{P}$ & ICZ & May-Aug \\
\hline \multicolumn{5}{|l|}{ Saxifragaceae Jussieu } \\
\hline Astilbe rivularis Buch.Ham ex D.Don & $\mathrm{S}$ & $\mathrm{P}$ & $\mathrm{TZ}$ & June-Nov \\
\hline \multicolumn{5}{|l|}{ Urticaceae Jussieu } \\
\hline Pilea pumila A.Gray & $\mathrm{H}$ & $\mathrm{P}$ & $\mathrm{TZ}$ & June-Aug \\
\hline Pilea scripta (Buch.Ham. ex D.Don) Wedd. & $\mathrm{H}$ & $\mathrm{P}$ & $\mathrm{TZ}$ & June-Aug \\
\hline Pilea ternifolia Wedd. & $\mathrm{H}$ & $\mathrm{P}$ & $\mathrm{TZ}$ & June-Aug \\
\hline \multicolumn{5}{|l|}{ Violaceae Batsch } \\
\hline Viola pumila Chaix & $\mathrm{H}$ & $A$ & $\mathrm{TZ}$ & April-June \\
\hline
\end{tabular}


Rai and Moktan; ARRB, 35(5): 63-81, 2020; Article no.ARRB.57913

\begin{tabular}{|c|c|c|c|c|}
\hline Taxa & Habit type & Life form & Distribution & Phenology \\
\hline \multicolumn{5}{|l|}{ Vitaceae Jussieu } \\
\hline Cayratia pedata (Lam.) Gagnep. & $\mathrm{CL}$ & $\mathrm{P}$ & TZ & June-Nov \\
\hline Cissus javana DC. & $\mathrm{CL}$ & $\mathrm{P}$ & $\mathrm{ICZ}$ & June-Oct \\
\hline \multicolumn{5}{|l|}{ Zingiberaceae Martinov } \\
\hline Cautleya spicata (Sm.) Baker & $\mathrm{H}$ & $\mathrm{P}$ & TZ & July-Nov \\
\hline Hedychium griffithianum Wall. & $\mathrm{H}$ & $\mathrm{P}$ & TZ & June-Nov \\
\hline \multicolumn{5}{|l|}{ Orchidaceae Jussieu } \\
\hline Acampe praemorsa var. praemorsa (Roxb.) Blatt. \& McCann & $\mathrm{H}$ & $\mathrm{P}$ & ICZ & March-April \\
\hline Acampe rigida (Buch.-Ham. ex Sm.) P.F.Hunt & $\mathrm{H}$ & $\mathrm{P}$ & $\mathrm{TZ}$ & Aug-Sept \\
\hline Aerides multiflora Roxb. & $\mathrm{H}$ & $\mathrm{P}$ & $\mathrm{TZ}$ & May-July \\
\hline Agrostophyllum myrianthum King \& Pantl. & $\mathrm{H}$ & $\mathrm{P}$ & $\mathrm{TZ}$ & July-Aug \\
\hline Agrostophyllum planicaule (Wall. ex Lindl.) Rchb.f. & $\mathrm{H}$ & $\mathrm{P}$ & $\mathrm{TZ}$ & July-Aug \\
\hline Bulbophyllum careyanum (Hook.) Spreng. & $\mathrm{H}$ & $\mathrm{P}$ & $\mathrm{TZ}$ & Nov \\
\hline Bulbophyllum crassipes Hook.f. & $\mathrm{H}$ & $\mathrm{P}$ & $\mathrm{TZ}$ & April \\
\hline Bulbophyllum odoratissimum (Sm.) Lindl.ex Wall. & $\mathrm{H}$ & $\mathrm{P}$ & $\mathrm{TZ}$ & April-Aug \\
\hline Bulbophyllum reptans (Lindl.) Lindl. ex Wall. & $\mathrm{H}$ & $\mathrm{P}$ & $\mathrm{TZ}$ & March \\
\hline Bulbophyllum roseopictum J.J.Verm., Schuit. \& de Vogel & $\mathrm{H}$ & $\mathrm{P}$ & $\mathrm{TZ}$ & Sept-Oct \\
\hline Coelogyne cristata Lindl. & $\mathrm{H}$ & $\mathrm{P}$ & $\mathrm{TZ}$ & May \\
\hline Coelogyne flaccida Lindl. & $\mathrm{H}$ & $\mathrm{P}$ & $\mathrm{TZ}$ & March \\
\hline Cymbidium aloifolium (L.) Sw. & $\mathrm{H}$ & $\mathrm{P}$ & $\mathrm{TZ}$ & Oct-Jan \\
\hline Cymbidium eburneum Lindl. & $\mathrm{H}$ & $\mathrm{P}$ & $\mathrm{TZ}$ & August \\
\hline Cymbidium elegans var. elegans Lindl. & $\mathrm{H}$ & $\mathrm{P}$ & $\mathrm{TZ}$ & August \\
\hline Cymbidium erythraeum Lindl. & $\mathrm{H}$ & $\mathrm{P}$ & $\mathrm{TZ}$ & May \\
\hline Dendrobium amoenum Wall. ex Lindl. & $\mathrm{H}$ & $\mathrm{P}$ & $\mathrm{TZ}$ & May-June \\
\hline Dendrobium aphyllum (Roxb.) C.E.C.Fisch. & $\mathrm{H}$ & $\mathrm{P}$ & $\mathrm{TZ}$ & Feb-July \\
\hline Dendrobium bicameratum Lindl. & $\mathrm{H}$ & $\mathrm{P}$ & $\mathrm{TZ}$ & April \\
\hline Dendrobium chrysanthum Wall. ex Lindl. & $\mathrm{H}$ & $\mathrm{P}$ & $\mathrm{TZ}$ & Sept \\
\hline Dendrobium comatum (Blumee) Lindl. & $\mathrm{H}$ & $\mathrm{P}$ & $\mathrm{TZ}$ & Sept \\
\hline Dendrobium denudans D.Don & $\mathrm{H}$ & $\mathrm{P}$ & $\mathrm{TZ}$ & Sept \\
\hline Dendrobium erythraeum Schuit. \& de Vogel & $\mathrm{H}$ & $\mathrm{P}$ & $\mathrm{TZ}$ & Sept \\
\hline Dendrobium humilicolle Schltr. & $\mathrm{H}$ & $\mathrm{P}$ & $\mathrm{TZ}$ & June \\
\hline Dendrobium longicornu Lindl. & $\mathrm{H}$ & $\mathrm{P}$ & $\mathrm{TZ}$ & June \\
\hline Dendrobium moschatum (Banks) Sw. & $\mathrm{H}$ & $\mathrm{P}$ & $\mathrm{TZ}$ & June \\
\hline Dendrobium pachyphyllum (Kuntze) Bakh.f. & $\mathrm{H}$ & $\mathrm{P}$ & $\mathrm{TZ}$ & March \\
\hline
\end{tabular}


Rai and Moktan; ARRB, 35(5): 63-81, 2020; Article no.ARRB.57913

\begin{tabular}{|c|c|c|c|c|}
\hline Taxa & Habit type & Life form & Distribution & Phenology \\
\hline Dendrobium transparens Wall. ex Lindl. & $\mathrm{H}$ & $\mathrm{P}$ & TZ & March \\
\hline Eria coronaria (Lindl.) Rchb.f. & $\mathrm{H}$ & $\mathrm{P}$ & $\mathrm{TZ}$ & May-June \\
\hline Gastrochilus calceolaris (Buch.-Ham. ex Sm.) D.Don & $\mathrm{H}$ & $\mathrm{P}$ & $\mathrm{TZ}$ & April-July \\
\hline Oberonia pachyrachis Rchb.f. ex Hook.f. & $\mathrm{H}$ & $\mathrm{P}$ & $\mathrm{TZ}$ & March-April \\
\hline Otochilus fuscus Lindl. & $\mathrm{H}$ & $\mathrm{P}$ & $\mathrm{TZ}$ & March \\
\hline Otochilus lancilabius Seidenf. & $\mathrm{H}$ & $\mathrm{P}$ & $\mathrm{TZ}$ & Oct-Nov \\
\hline Pleione praecox (Sm.) D.Don & $\mathrm{H}$ & $\mathrm{P}$ & $\mathrm{TZ}$ & Sept-Oct \\
\hline Porpax elwesii (Rchb.f.) Rolfe & $\mathrm{H}$ & $\mathrm{P}$ & $\mathrm{TZ}$ & June \\
\hline Porpax filiformis (Wight) Schuit., Y.P.Ng \& H.A.Pedersen & $\mathrm{H}$ & $\mathrm{P}$ & $\mathrm{TZ}$ & Dec \\
\hline Rhynchostylis retusa (L.) Blume & $\mathrm{H}$ & $\mathrm{P}$ & $\mathrm{TZ}$ & Nov \\
\hline Thunia alba (Lindl.) Rchb. & $\mathrm{H}$ & $\mathrm{P}$ & $\mathrm{TZ}$ & June \\
\hline Uncifera obtusifolia Lindl. & $\mathrm{H}$ & $\mathrm{P}$ & $\mathrm{TZ}$ & June \\
\hline Vanda cristata Wall.ex Lindl. & $\mathrm{H}$ & $\mathrm{P}$ & $\mathrm{TZ}$ & May-June \\
\hline Vandopsis undulata (Lindl.) J.J.Sm. & $\mathrm{H}$ & $\mathrm{P}$ & $\mathrm{TZ}$ & May-June \\
\hline \multicolumn{5}{|l|}{ Aspleniaceae Newman } \\
\hline Asplenium ensiforme Wall. ex Hook. \& Grev & $\mathrm{H}$ & $\mathrm{P}$ & TZ & June-Sept \\
\hline Asplenium phyllitidis D.Don & $\mathrm{H}$ & $\mathrm{P}$ & $\mathrm{TZ}$ & June-Sept \\
\hline Asplenium yoshinagae subsp. indicum (Sledge) Fraser-Jenk. & $\mathrm{H}$ & $\mathrm{P}$ & $\mathrm{TZ}$ & March-April \\
\hline \multicolumn{5}{|l|}{ Lindsaeaceae M. R. Schomb } \\
\hline Lindsaea orbiculata (Lam.) Mett. ex Kuhn & $\mathrm{H}$ & $\mathrm{P}$ & TZ & July-Oct \\
\hline \multicolumn{5}{|l|}{ Lycopodiaceae P. Beauvois ex Mirbel } \\
\hline Huperzia pulcherrima (Wall. ex Hook. \& Grev.) Pic.Serm & $\mathrm{H}$ & $\mathrm{P}$ & ICZ & July-Oct \\
\hline Huperzia squarrosa (G.Forst.)Trevis & $\mathrm{H}$ & $\mathrm{P}$ & $\mathrm{ICZ}$ & July-Oct \\
\hline \multicolumn{5}{|l|}{ Nephrolepidaceae Pichi Sermolli } \\
\hline Nephrolepis cordifolia (L.) C.Presl & $\mathrm{H}$ & $\mathrm{P}$ & TZ & Aug-Nov \\
\hline \multicolumn{5}{|l|}{ Polypodiaceae J. Presl \& C. Presl } \\
\hline Drynaria mollis Bedd. & $\mathrm{H}$ & $\mathrm{P}$ & $\mathrm{TZ}$ & June-Aug \\
\hline Drynaria propinqua(Wall. ex Mett.) J.Sm. ex Bedd. & $\mathrm{H}$ & $\mathrm{P}$ & $\mathrm{TZ}$ & June-Aug \\
\hline Elaphoglossum callifolium (Blume) J.Sm. & $\mathrm{H}$ & $\mathrm{P}$ & $\mathrm{TZ}$ & May-July \\
\hline Goniophlebium amoenum (Wall. ex Mett.) Bedd. & $\mathrm{H}$ & $\mathrm{P}$ & $\mathrm{TZ}$ & Aug-Sept \\
\hline Goniophlebium argutum J.Sm & $\mathrm{H}$ & $\mathrm{P}$ & $\mathrm{TZ}$ & Aug-Sept \\
\hline Goniophlebium lachnopus (Wall. ex Hook.) J.Sm & $\mathrm{H}$ & $\mathrm{P}$ & $\mathrm{TZ}$ & Aug-Sept \\
\hline Lepisorus scolopendrium (Buch-Ham ex D.Don) Mehra \& Bir & $\mathrm{H}$ & $\mathrm{P}$ & $\mathrm{TZ}$ & July-Sept \\
\hline Leucostegia truncata (D.Don) Fraser-Jenk. & $\mathrm{H}$ & $\mathrm{P}$ & $\mathrm{TZ}$ & July-Sept \\
\hline
\end{tabular}


Rai and Moktan; ARRB, 35(5): 63-81, 2020; Article no.ARRB.57913

\begin{tabular}{|c|c|c|c|c|}
\hline Taxa & Habit type & Life form & Distribution & Phenology \\
\hline Loxogramme cuspidata (Zenker) M.G.Price & $\mathrm{H}$ & $\mathrm{P}$ & $\mathrm{TZ}$ & June-Sept \\
\hline Loxogramme involuta (D.Don) C.Presl & $\mathrm{H}$ & $\mathrm{P}$ & $\mathrm{TZ}$ & Aug-Oct \\
\hline Microsorum membranaceum (D.Don) Ching & $\mathrm{H}$ & $\mathrm{P}$ & $\mathrm{TZ}$ & Aug-Oct \\
\hline Oleandra pistillaris (Sw.) C.Chr. & $\mathrm{H}$ & $\mathrm{P}$ & $\mathrm{TZ}$ & Aug-Oct \\
\hline Oleandra wallichii (Hook.) C.Presl & $\mathrm{H}$ & $\mathrm{P}$ & $\mathrm{TZ}$ & Aug-Oct \\
\hline Phymatosorus cuspidatus (D.Don) Pic.Serm & $\mathrm{H}$ & $\mathrm{P}$ & $\mathrm{ICZ}$ & Aug-Oct \\
\hline Pyrrosia costata (Wall. ex C.Cresl) Tagawa \& K.Iwats. & $\mathrm{H}$ & $\mathrm{P}$ & ICZ & June-Sept \\
\hline Pyrrosia lanceolata (Wall.) Farw. & $\mathrm{H}$ & $\mathrm{P}$ & ICZ & June-Sept \\
\hline Pyrrosia lingua (Thunb.) Farw. & $\mathrm{H}$ & $\mathrm{P}$ & $\mathrm{ICZ}$ & June-Sept \\
\hline Pyrrosia manni (Giesenh.) Ching & $\mathrm{H}$ & $\mathrm{P}$ & ICZ & June-Sept \\
\hline Pyrrosia nuda (Giesenh.) Ching & $\mathrm{H}$ & $\mathrm{P}$ & $\mathrm{ICZ}$ & June-Sept \\
\hline Selliguea oxyloba (Wall. ex Kunze) Fraser-Jenk. & $\mathrm{H}$ & $\mathrm{P}$ & $\mathrm{TZ}$ & Aug-Oct \\
\hline \multicolumn{5}{|l|}{ Pteridaceae E.D.M. Kirchner } \\
\hline Haplopteris elongata (Sw.) E.H. Crane & $\mathrm{H}$ & $\mathrm{P}$ & $\mathrm{ICZ}$ & July-Oct \\
\hline Vittaria sp. & $\mathrm{H}$ & $\mathrm{P}$ & $\mathrm{TZ}$ & July-Sept \\
\hline
\end{tabular}




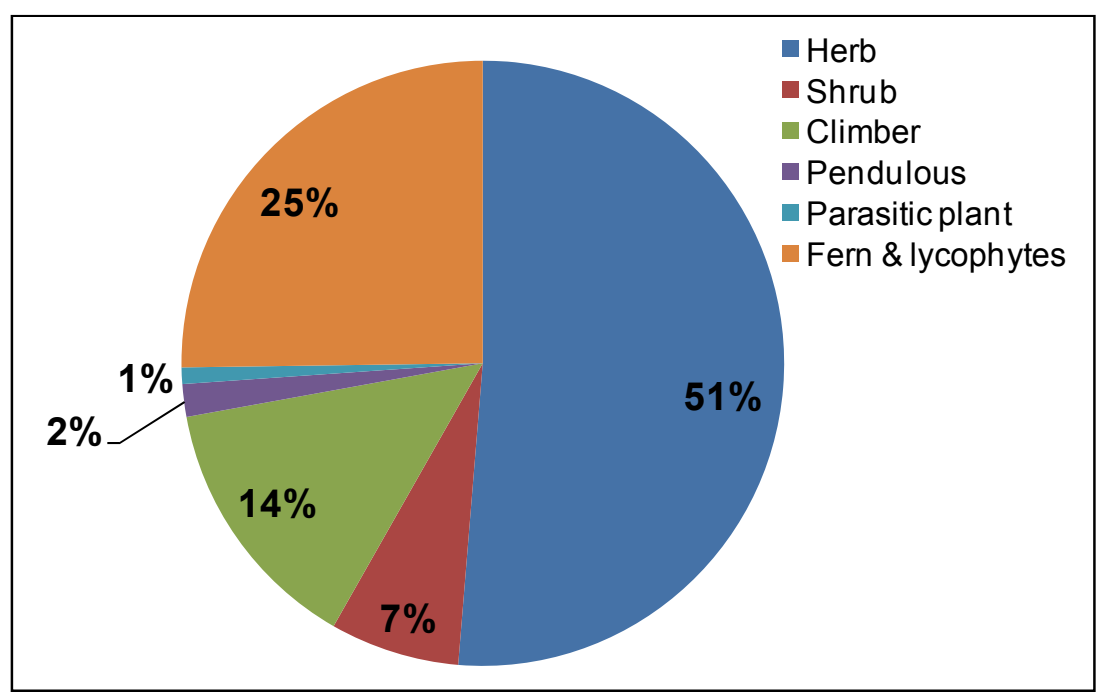

Fig. 4. Different habit groups of vascular epiphytes

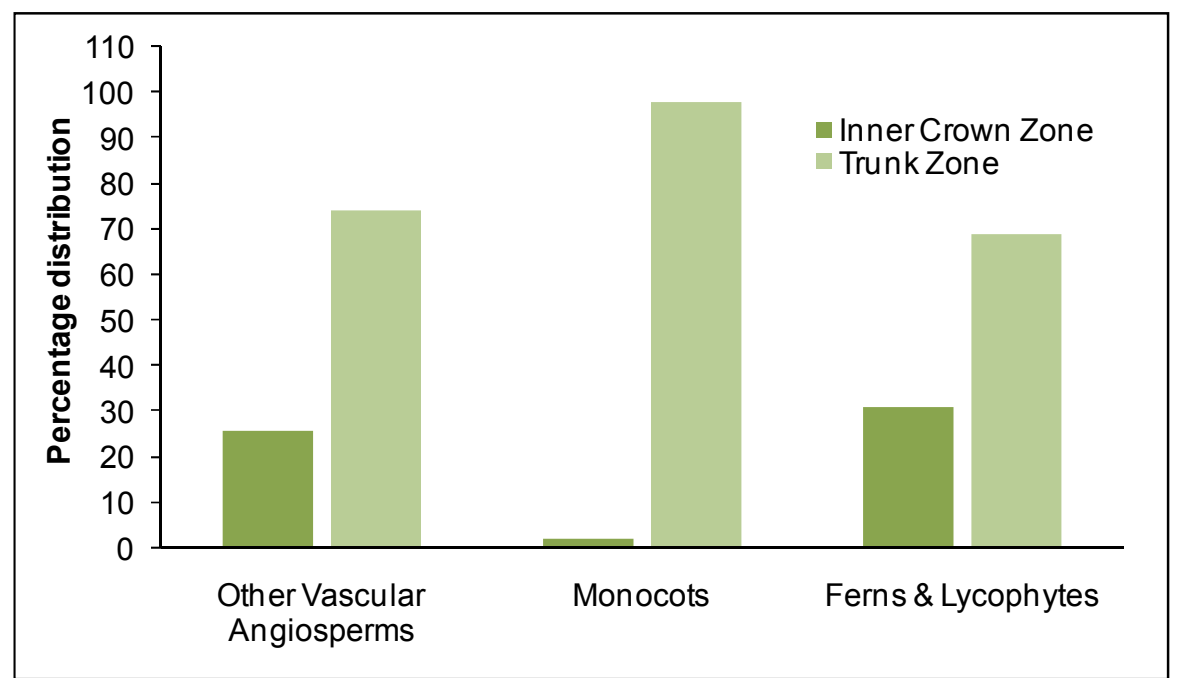

Fig. 5. Percentage distribution in host tree trunk

\subsubsection{Microhabitat and phenology}

The entire ferns and lycophytes including Orchidaceae recorded from the study area were found to be perennial, while among the other vascular angiosperms, $89 \%$ were perennial while only $11 \%$ of species were found to be annual. All the epiphytic angiosperms were found to be facultative that grew either on the host tree trunk or as terrestrial, except species of Aeschynanthus which is truly epiphytic. Ferns and lycophytes are divided into three categories a) Epiphyte/Terrestrial fern that grew both on tree trunk and forest floor representing $59 \%$ b) Epiphyte/Epilithic that grew on tree trunk and as lithophytes represented $31 \%$ and c) True epiphyte that grew only on host tree trunk was $10 \%$. Similarly, Orchidaceae were also categorized into a) True epiphyte, growing only on the host tree trunk representing $41 \%$ and b) Epiphyte/Epilithic with $59 \%$ that grew both on the tree trunk as well as on the rock surface.

In the present study, the phenology of the taxa that was observed, highlighted $24 \%$ of other vascular angiosperms bloomed in spring and summer season whereas $60 \%, 9 \%$ and $7 \%$ flowered in monsoon, autumn and winter season respectively. Similarly, $46 \% \quad(19$ taxa) of Orchidaceae bloom in spring while $27 \%, 22 \%$ 
and $5 \%$ flowered in summer, autumn and winter season respectively (Fig. 6). It can be concluded that maximum number of other vascular angiosperms flower in wet monsoon season whereas spring season was observed to be most preferable flowering period for Orchidaceae.

\subsection{Discussion}

The present study recorded a number of vascular epiphytic taxa that were distributed within a short range between sub-temperate to temperate forests of Darjeeling Himalaya. Similar type of works from different regions includes sampling vascular epiphyte diversity, species richness, community structure tree line-composition and population biology have also been conducted $[57,58,59]$.

Association of epiphytes excluding orchids to host and environmental variables were highlighted [44] whereas in the present study, all the epiphytes including Orchidaceae were related to host and environmental variables. Previous study in Darjeeling Himalaya [46] recorded 88 epiphytic species in upper montane tropical forest which was lesser than the present study that identified 115 vascular epiphytic species. The study strongly supports the biggest abundance of Orchidaceae in the area and the distribution of different epiphytic species closer to the first branching. Some of the recorded epiphytic angiosperms in the present study such as Neohymenopogon parasiticus, Vaccinium retusum, Lysionotus serratus, Hoya linearis, Peperomia heyneana, Polygonatum oppositifolium were recorded more than 50 years ago in the region [45]. Though India's epiphytic orchid is to be found primarily in the Eastern Himalayas and Western Ghats, 99 epiphytic orchids in Western Himalayas have also been recorded [40] which was however less than the present documentation. Earlier study on fern and fern allies from Western Ghats [39] identified many epiphytes common to the fern and fern allies of Darjeeling Himalayas and some of them have also been identified in this communication too. Epiphytic species richness increases with increasing rainfall [60] while [61] found a decrease of epiphytic richness in the most humid habitats. [62] documented the massive decline of epiphyte diversity at high elevation upto $4000 \mathrm{~m}$ and the actual mechanism of limited epiphytic growth at low temperature are still unknown. Ferns are the most common vascular epiphytes in humid temperate regions [63] while in the present study Orchidaceae was the most abundant vascular epiphytes.

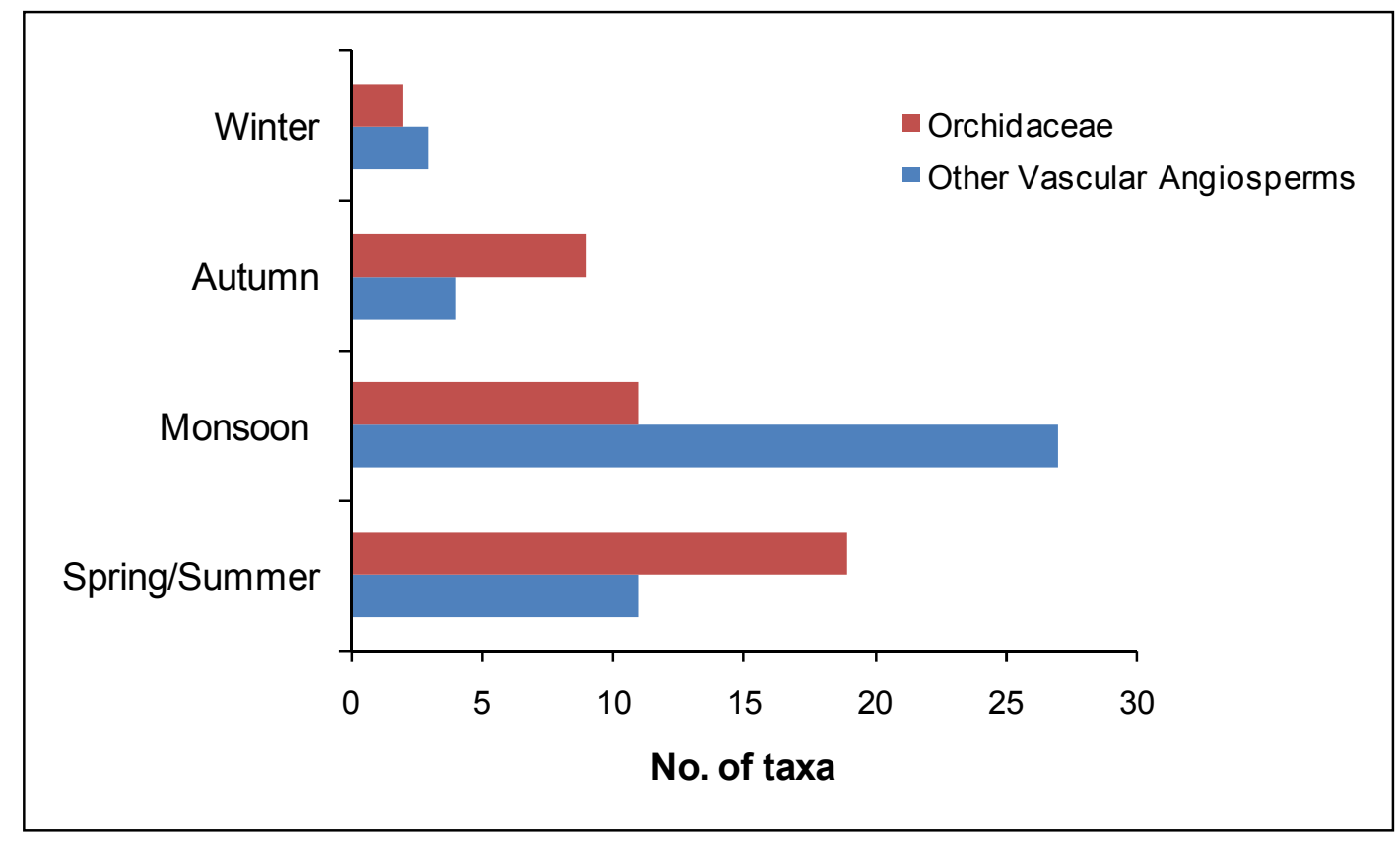

Fig. 6. Phenological status for the epiphytic taxa 

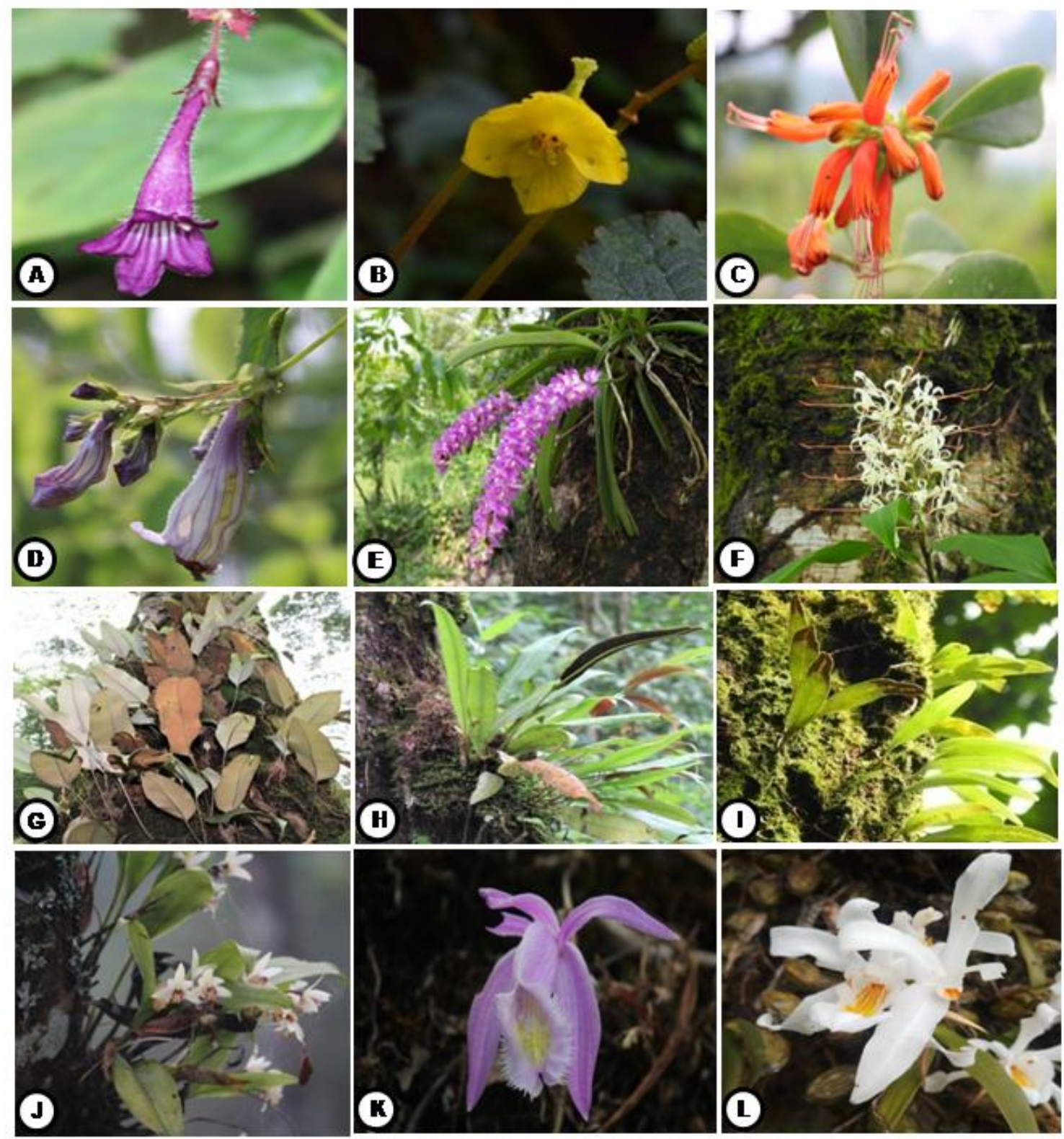

Plate 1. A. Didymocarpus aromaticus B. Begonia flaviflora C. Aeschynanthus hookeri D. Lysionotus serratus E. Rhynchostylis retusa F. Hedychium griffithianum G. Pyrrosia lingua H. Elaphoglossum callifolium I. Pyrrosia lanceolata J. Eria coronaria K. Pleione praecox L. Coelogyne cristata

Host tree interaction in nine species harboring eleven epiphytic taxa have been recorded from agro forestry system [64]. Our study reflects the decrease in the number of vascular epiphytes from trunk zone to crown zone. This decrease in epiphytic richness may be due to increase in ultraviolet radiation, decrease in humidity and increase in photon flux density along the increasing canopy height of the hosts [65], lack of adaptation for high water stress in vertical canopy branches $[66,67,68]$.

Parasitic plant like Dendrotrophe granulata (Hook. f. \& Thomson ex A.DC.) Henry \& Roy and some critically threatened (CR) orchid Gastrochilus calceolaris (Buch.-Ham. ex Sm.) D.Don [69] species were also recorded from study area including Cymbidium eburneum Lindl. 
which is endemic to Eastern Himalaya and Northeastern India [70]. Species like Agapetes incurvata (Griff.) Sleumer was found to be endemic to eastern Himalaya while Thunbergia lutea T.Anderson is endemic to Darjeeling region. Many orchid taxa Agrostophyllum myrianthum King \& Pantl., Bulbophyllum reptans (Lindl.) Lindl. ex Wall., B. odoratissimum (Sm.) Lindl., Pleione praecox (Sm.) D.Don, Vandopsis undulate (Lindl.) J.J.Sm. are sparsely populated and Aerides multiflora Roxb., Cymbidium erythraeum Lindl., Dendrobium bicameratum Lindl., Gastrochilus calceolaris (Buch.-Ham. ex
Sm.) D.Don, Porpax elwesi (Rchb.f.) Rolfe, Unifera obtusifolia Lindl., are rarely found while Agrostophyllum planicaule (Wall. ex Lindl.) Rchb.f. (NT), Bulbophyllum tortuosum (Blume) Lindl. (NT), Cymbidium eburneum Lindl., (NT), are categorized as near threatened [71]. Species like Holboellia angustifolia Wall., Elaphoglossum callifolium (Blume) J.Sm., Hoya serpens (Hook.f.), Polygonatum oppositifolium (Wall.) Royle, Begonia flaviflora H. Hara, Herpetospermum tonglense (C.B.Clarke) H.Schaef. \& S.S.Renner, were found to be less in population number.
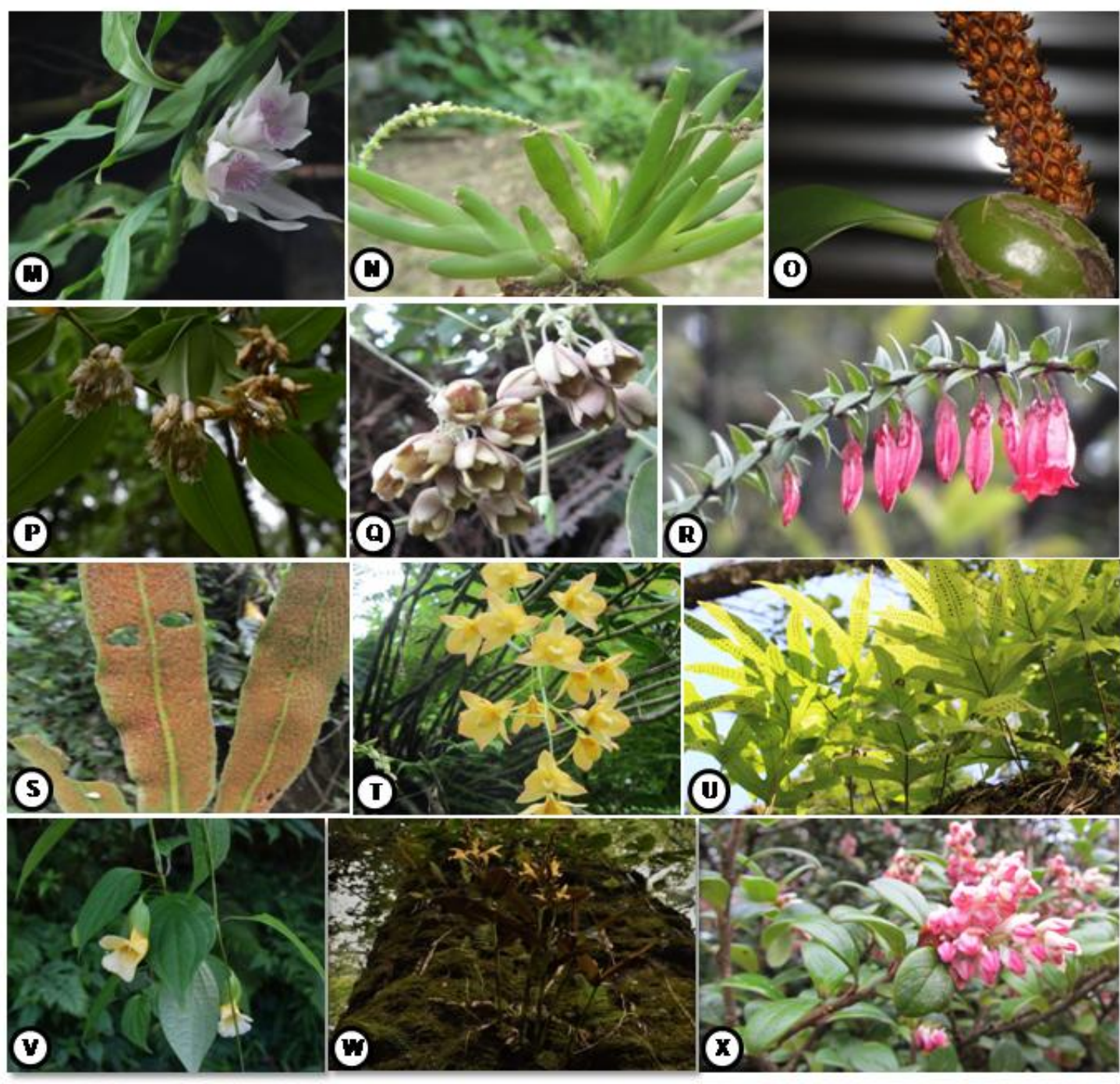

Plate 2. M. Thunia alba N. Oberonia pachyrachis O. Bulbophyllum crassipes P. Polygonatum oppositifolium Q. Holboellia angustifolia R. Agapetes serpens S. Pyrrosia costata T. Dendrobium moschatum U. Selliguea oxyloba V. Thunbergia lutea W. Cautleya spicata $X$. Vaccinium retusum 
The availability of enough time and space for colonization and higher diversity of microhabitats are the main reason for more epiphytic species on large trees rather than smaller trees $[72,73$, 74]. In the present investigation, maximum individual count of taxa was recorded from the old trees with larger trunk $>100 \mathrm{~cm} \mathrm{CBH}$ whereas only few individuals were recorded from trees having smaller trunk with $<40 \mathrm{~cm} \mathrm{CBH}$. Hence there is positive relationship between phorophyte size and abundance of vascular epiphytes which supports the findings of other studies $[33,75,76]$. Similarly host bark traits are found to affect the distribution and development of vascular epiphytes, rough or coarse bark of host tree support many epiphytes whereas host with smooth bark supported few epiphytes [77] because they retain moisture for longer and seedling recruitment is better in rough bark than on smooth bark [42].

Although a good number of taxa have been observed in the study, the plants are still under serious threat. Being an extension of Himalaya hotspot it is not difficult to imagine the extent of anthropogenic pressure exerted on natural vegetation almost in all corners of the area. Habitat loss, forest destruction and degradation, loss of suitable host tree and human impact such as settlement and agriculture are the greatest threat for epiphytes [41]. Human population and the rate of habitat modification is too much in the hill region and almost all forests are affected. The collections of plants for ethnobotanical practices by the inhabitants living in the vicinity of subtemperate forests are also one of the reasons for decline in the species. Some ethno-medicinal plants have also been identified that are widely used. Taxa like Astilbe rivularis was commonly used against ulcer, bleeding during child birth, asthma and brain diseases [78]. Similarly, rhizome juice of Cautleya spicata was used in the treatment of stomach disorders [79]. Among the orchids that were recorded, species that attributed to medicinal properties against various ailments include Acampe praemorsa (Antirheumatism) [80], Aerides multiflora (Antibacterial), Dendrobium chrysanthum (antiinflamatory activity) and Coelogyne cristata (Phytoalexin) [81]. Beside these, indiscriminate use of natural resources including forest fuels, collection of medicinal plants and ornamental orchids for house nursery and commercial marketing are inevitable reasons for the decline in the diversity of epiphytes as well as other taxa. It has also come to notice that the frond of Nephrolepis cordifolia is being extensively collected by the local people leading to the decrease in species abundance at some sites under study.

\section{CONCLUSION}

The vascular epiphytic flora of sub-temperate region of Darjeeling Himalaya is valuable natural resources. The present investigation resulted in documenting a total of 115 other vascular angiosperms, ferns and Orchidaceae where the other vascular angiosperms preferred wet monsoon for flowering as compared to spring season mostly favoured by Orchidaceae. The host tree reflected dominance of trunk zone over inner crown zone with respect to epiphytic species distribution. This work would definitely provide significant information for regional flora as well as flora of eastern Himalaya hotspot. However, it has been observed that deforestation, collection of plants for commercial purposes, construction and agriculture practices prevailing in the study area have been causing frequent change in species diversity and composition of vascular epiphytes and other taxa as well. Many of the taxa are already under low population count. Therefore there lies role of conservationist, taxonomist, ecologist, in collaboration with forest departments and concerned participation of the locals for proper conservation of the rich and valuable epiphytic taxa in the region.

\section{ACKNOWLEDGEMENTS}

The first author is grateful to University Grant Commission, New Delhi, India for financial assistance. The authors are also thankful to the Forest Department, Government of West Bengal for all the necessary permissions.

\section{COMPETING INTERESTS}

Authors have declared that no competing interests exist.

\section{REFERENCES}

1. Jacques MA, Morris CE. A review of issues related to the Quantification of bacteria from the phyllosphere. FEMS Microbiology Ecology. 1995;18(1):1-14.

2. Heywood $\mathrm{VH}$. Flowering plants of the world. Oxford University Press. New York; 1993. 
3. Fattland B. Vascular epiphytes of central Veracruz, Mexico. Jour of Vegetable Sci. 1996;6:719-728.

4. Benzing DH. Vascular Epiphytes. Forest Canopise. In: Margaret DL, H Bruce R (eds). Elsevier Academic Press, San Diego; 2004.

5. Hietz P. Conservation of vascular epiphyte diversity in Mexican coffee plantations. Conservation Biology. 2005;19:391-399.

6. Zhang Z, Yan Y, Tian Y. Distribution and conservation of orchid species richness in China. Biological Conservation. 2015;181: 64-72.

7. Getaneh ZA, Gamo FW. Vascular epiphytes in Doshke and Kurpaye: A comparative study, Gamo Gofa, Ethiopia. Inter Jour of Biodiversity. 2016;1-10.

8. Kress WJ. The systematic distribution of vascular epiphytes. In: Lüttge U (Eds.) Vascular Plants as Epiphytes Evolution and Ecophysiology. Springer-Verlag, Berlin; 1989.

9. Benzing DH. Vascular Epiphytes. Cambridge University Press, USA; 1990.

10. Kress WJ. The systematic distribution of vascular epiphytes: An update. Selbyana. 1986;9(1):2-22.

11. Benzing DH. Vascular epiphytes. Cambridge University press, USA; 2004.

12. Alataş $M$, Batan $N$, Ezer $T$. The epiphytic bryophyte vegetation of Kamilet Valley (Artvin, Turkey). Turk J of Botany. 2019;49: 1779-1786.

13. Gentry $\mathrm{AH}$, Dodson C. 1987. Contribution of non trees to species richness of a tropical rain forest. Biotropica. 1987;19(2): 149-156.

14. Benzing DH. Vulnerabilities of tropical forests to climate change: The significance of resident epiphytes. Climatic Change. 1998;39(2-3):519-540.

15. Zotz G. Vascular epiphytes in the temperate zones - A review. Plant Ecology. 2005;176(2):173-183.

16. Gotsch SG, Nadkarni N, Amici A. The functional role of epiphytes and aboreal site in tropical montane forests. Jour of Trop Eco. 2016;1:1-14.

17. Shanavaskhan AE, Sivadasan M, Alfarhan $\mathrm{AH}$, Thomas J. Ethnomedicinal aspects of angiospermic epiphytes and parasites of Kerela, India. Indian Jour of Trad Know. 2012;11(2):250-258.
18. Turner IM, Tan HTW, Wee YC, Ibrahim $A B$, Chew PT. A study of plant species extinction in Singapore: lessons for the conservation of tropical biodiversity. Conservation Biology. 1994;8(3):705-712.

19. Sanger JC, Kirkpartrick JB. Patterns of morphological and physiological traits of epiphytes within trees and between elevations in subtropical Australian rainforest. Cunninghamia. 2017;17:15-25.

20. Hietz $P$, Briones $O$. Correlation between water relations and within-canopy distribution of epiphytic ferns in a Mexican cloud forest. Oecologia. 1998;114:305316.

21. Higgins WE. Orchid adaptations to an epiphytic lifestyle. In: Lowman MDR, Rinker HB (Eds.), Forest Canopies. Elsevier Academic Press, London (UK); 2004.

22. Reyes G, Chang MM, Jones GD, Griffiths $\mathrm{H}$. Water vapour isotope exchange by epiphytic bromeliads in tropical dry forests reflects niche differentiation and climatic signals. Plant, cell and environment. 2008; 31(6):828-84.

23. Triana-Moreno LA, Garzon-Venegas NJ, Sanchez-Zambrano J, Vargas O. Epıfitas vasculares como indicadores deregeneracio'n en bosques intervenidos de la amazo^nia Colombiana. Acta Biologica Colombiana. 2003;8:31-42.

24. Zotz G, Bader M. 2009. Epiphytic plants in a changing world: global change effects on vascular and non-vascular epiphytes. Progress in Botany. 2009;70:147-170.

25. Richter M. Methoden der Klimaindikation durch pflanzenmorphologische Merkmale in den Kordilleren der Neotropis. Erde. 1991;122:267-289.

26. Lugo AE, Scatena FN. Epiphytes and climate change researchin the Caribbean: A proposal. Selbyana. 1992;13:123-130.

27. Barthlott W, Schmit-Neuerburg V, Nieder J, Engwald S. Diversity and abundance of vascular epiphytes: A comparison of secondary vegetation and primary montane rain forest in the Venezuelan Andes. Plant Ecology. 2001;152(2):145156.

28. Clavel J, Julliard R, Devictor V. Worldwide decline of specialist species: Toward a global functional homogenization? Frontier in Ecol and the Environ. 2011;9:222-228. 
29. Colwell RK, Dunn RR, Harris NC. Coextinction and persistence of dependent species in a changing world. Annual Review of Ecol, Evol and Syst. 2012;43: 183-203.

30. Kromer T, Gradstein SR. Vascular epiphytes. Biodiversity Sampling Protocol. 2016;26-36.

31. Arevalo R, Betancur J. Vertical distribution of vascular epiphyes in four forest types of the Serrania De Chiribiquete, Colombian Guayana. Selbyana. 2006;27(2):175-185.

32. Ezer T, Kara R. Succession of epiphytic bryophytes in Cedrus libani forest on the Meydan Plateau (Aladag). Turkish Jour of Botany. 2013;37:389-397.

33. Brown M, Mariscal A, Chinchero MA, Diaz A. Biotic factors affecting the abundance of vascular epiphytic bromeliads growing in cloud forest in Reserva Biologica Los Cedros, Ecuador. Annual Research \& Review in Biology. 2015;6(6):355-363.

34. Annaselvam J, Parthasarathy N. 2001. Diversity and distribution of herbaceous vascular epiphytes in a tropical evergreen forest at Varagalaiar, Western Ghats, India. Biodiversity Conservation. 2001;10 (3):317-329.

35. Mathew J. Epiphytic angiosperm of Achankovil forests, Southern western Ghats, kerela, India. Inter Jour of Sci Res. 2012;2(11)240-242.

36. Kumar MSM. Studies on the epiphytic flora in the tropical forest ecosystem of Western Ghats with special reference to Nilgiri Biosphere Reserv. KFRI Research Report. 1998;1-141.

37. Tewari M, Upreti N, Pandey P, Singh SP. Epiphytic succession on tree trunks in a mixed oak-cedar forest, Kumaun Himalaya. Vegetation. 1985;63:105-112.

38. Samant SS, Rawat RS, Dhar U. Epiphytic orchids of Askot Wildlife sanctuary in Kumaun Himalay, India: Conservationa imperatives. Environ Conser. 1995;22(1): 71-74.

39. Awasthi DK, Sharma MP. Ecological and phytogeographical observations on the ferns and fern-allies of Nagur block (Chamoli Garhwal), Western Himalayas. Proc Indian Aca Sci. 1980;89(4):307-313.

40. Jalal JS. Distribution pattern of orchids in Uttarakhand, Western Himalayas, India. Inter Jour of Plant Bio. 2012;3(5):24-26.
41. Adhikari YP, Fischer A, Fischer HS. Micosite conditions of epiphytic orchids in a human impact gradient in Kathmandu Valley, Nepal. Jour of Moun Sci. 2012;9: 331-342

42. Timsina B, Rokaya MB, Munzbergova Z, Kindlmann $P$, Shrestha B, Bhattarai B, Raskoti BB. Diversity, distributon ans hostspecies association of epiphytic orchids in Nepal. Biodivers Conserv. 2016;1-18.

43. Bhattarai KH, Vetaas OR, Grytnes JA. Fen species richness along a central Himalayan elevational gradient, Nepal. Jour of Biogeo. 2004;31:389-400.

44. Padmawathe R, Qureshi Q, Rawat GS. Effects of selective logging on vascular epiphyte diversity in a moist lowland forest of Eastern Himalaya, India. Biological Conservation. 2004;119:81-92.

45. Sen GC. The epiphytic flowering plants of Darjeeling Hills, other than Orchids. Nelumbo. 1963;5(2).

46. Ghosh A, Saha S. Distribution of epiphytes, parasites and mistletoes on six species of Phorophytes in upper montane tropical forest of Darjeeling, West Bengal. Indian Jour of Appl and Pure Biology.

47. Nugraha AS, Triatmoko B, Wangchuk $P$, Keller. Vascular epiphytic medicinal plants as sources of therapeutic agents:their ethnopharmacological uses, Chemical Composition and Biological activities. Biomolecules. 2020;10(2):181.

48. Gamble JS. List of the trees, shrubs and large climbers found in the Darjeeling District. Bengal, ed. 2, Calcutta; 1896.

49. Hooker JD. Himalayan Journals I. London; 1954.

50. Biswas KP. Plants of Darjeeling and Sikkim Himalayas. Govt. of West Bengal, India; 1966.

51. Bhujel RB. Studies on the dicotyledonous flora of Darjeeling district. PhD. Thesis, University of North Bengal, West Bengal, India; 1996.

52. Das AP. Floristic studies in Darjeeling Hills. Bulletin of the Bot Survey of India. 2004; 46:1-4.

53. Das AP. Diversity of the Angiospermic flora of Darjeeling Hills. Taxonomy and Biodiversity (ed. AK Pandey), CBS, Publishers. New Delhi. 1995;118-127.

54. Rai U. Characterization of Plant Biodiversity in Darjiling hills using Remote 
Sensing techniques, Ph.D. Thesis, North Bengal University; 2006.

55. Jain SK, Rao RR. A Handbook of field and Herbarium Methods. Today and tomorrow's Printers and Publishers, New Delhi; 1977.

56. POWO 2020. Plants of the world online. Facilitated by the Royal Botanic Gardens, Kew.

Available:http://www.plantsoftheworldonlin e.org

57. Zotz G, Bader MY. Sampling vascular epiphytic diversity-species richness and community structure. Ecology. 2011;17: 103-112.

58. Zotz G, Leiva GM, Wagner K. Vascular epiphytes at the treeline composition of species assemblages and population biology. Flora. 2014;209:385390.

59. Mondragon D, Valverde T, Hernandez AM. 2015. Population ecology of epiphytic angiosperms: A review. Tropical Ecol. 2015;56(1):01-39.

60. Kreft H, Koster N, Kuper W, Nieder J. Diversity and biogeography of vascular epiphytes in Western Amazonia, Yasun?? Ecuador. Jour of Biogeo. 2004;31(9):14631476.

61. Wolf JHD, Alejandro FS. Patterns in species richness and distribution of vascular epiphytes in Chiapas, Mexico. Jour of Biogeo. 2003;30:1689-1707.

62. Kromer T, Kessler M, Gradstein SR, Acebey A. Diversity patterns of vascular epiphytes along an elevational gradient in the Andes. Jour of Biogeo. 2005;32:17991809.

63. Zotz G. The role of the vascular epiphytes in the ecosystem. In: Plants on Plants. The biology of vascular epiphytes. Fascinating Ife scence. Springer Cham; 2016.

64. Ayele D, Awas T. Ecology of vascular epiphytic plants in Gedeo Agro-forestry System, Southern Ethiopia. Inter J of Current Research. 2018;10(2):6485064854.

65. Wang X, Long W, Schamp BS, Yang X, Kang $Y$. Vascular epiphyte diversity differs with host crown zone and diameter but not orientation in a tropical cloud forest. Plos One. 2016;11(7):1-13.

66. Laman TG. Ficus stupenda germination and seedling establishin a Bornean rain- forest canopy. Ecology. 1995;76:26172626.

67. Mantovani A. Leaf morpho-physiology and distribution of epiphytic aroids along a vertical gradient in a Brazilian rain forest. Selbyana. 1999;20.

68. Patino S, Gilbert GS, Zotz G, Tyree MT. Growth and survival of aerial roots of hemiepiphytes in a lower montane tropical moist forest in Panama. Jour of Tropical Ecol. 1999;15:651-665.

69. Agoo EMG, Cootes J, Golamco AJr and deVogel EF, Tiu D. Gastrochilus calceolaris. The IUCN Red List of Threatened Species; 2004.

70. Gogoi K, Kumaria S, Tandon P. Ex situ conservation of Cymbidium eburneum Lindl: A threatened and vulnerable orchid by asymbiotic seed germination. Biotech. 2012;2:337- 343.

71. Yonzone R. Exact field status, Habitat and local distribution of Orchid species of Darjeeling Himalaya of West Bengal, India. Advances in Agric Tech and Plant Sci. 2019;2(1):1-34.

72. Neider J, Prosperi J, Michaloud G. Epiphytes and their contribution to canopy diversity. Plant Ecol. 2001;153:51-63.

73. Flores-Palacios A, Garcia-Franco J. Effect of the structure and nutrient content of oak epiphyte communities. Plant Ecol. 2004; 173:259-269.

74. Wang Q, Gaun WB, Wong MHG, Ranjitkar S, Sun WN, Pan Yao et al. Tree sixe predicts the vascular epiphytic richness of traditional cultivated tea plantations in Southwestern China. Global Ecol and Conser. 2017;10:147-153.

75. Catling PM, Lefkovitch LP. Associations of vascular epiphytes in a Guatemalan cloud forest. Biotropica. 1989;21:35-40.

76. Migenis LE, Ackerman JD. Orchid phorophyte relationships in a forest watershed in Puerto Rico. J. Trop. Ecol. 1993;9:231-240.

77. Wyse SV, Burns BR. Do host bark traits influence trunk epiphyte communities? New Zealand Jour of Eco. 2011;35(3):296301.

78. Hussain S, Hore DK. Collection and conservation of major medicinal plants of Darjeeling and Sikkim Himalayas. Indian Jour of Traditional Know. 2007;6(2):352357. 
79. Choudhary RK, Lee J. A floristic reconnaissance of Churdhar Wildlife sanctuary of Himachal Pradesh, India. Manthan Intern Jornal. 2012;13:2-12.

80. Vibha S, Hebbar SS, Mahalakshmi SN, Kekuda TRP. A comprehensive review on ethnobotanical applications and pharmacological activities of
Acampe praemorsa (Roxb.) Blatt. \& McCann (Orchidaceae). Jour of Drug Delivery and Therapeutics. 2019;9(1):331336.

81. Singh S, Singh AK, Kumar S, Kumar M, Pandey PK. Medicinal properties and uses of orchids: A concise review. Elixir Appl Botany. 2012;52:11627-11634.

(c) 2020 Rai and Moktan; This is an Open Access article distributed under the terms of the Creative Commons Attribution License (http://creativecommons.org/licenses/by/4.0), which permits unrestricted use, distribution, and reproduction in any medium, provided the original work is properly cited.

Peer-review history:

The peer review history for this paper can be accessed here:

http://www. sdiarticle4.com/review-history/57913 